\title{
The Effect of Process Condition on the Structure and Optical Properties of $\mathrm{Zn} / \mathrm{Fe} / \mathrm{Sn} / \mathrm{Sb}-\mathrm{TiO}_{2}$ Three Dimensional Particle-Electrode
}

\author{
Shuxia Gao ${ }^{1 \mathrm{a}}$, Deyi Wang ${ }^{2 \mathrm{~b}}$,Peng Zhang ${ }^{2 \mathrm{c}}$, Xianjun Guo ${ }^{2 \mathrm{~d}}$ and Xiaolong Zhang ${ }^{2 \mathrm{e}}$ \\ ${ }^{1}$ School of Opt-electric Information Technology, Yantai University, Yantai China 264005 \\ ${ }^{2}$ School of Environment and Material Engineering, Yantai University, Yantai China 264005 \\ agaoshux@126.com, bwangdy107@163.com, ${ }^{\mathrm{c} p p 315618 @ 163 . c o m, ~}{ }^{\mathrm{d} x j g 10 @ 163 . c o m, ~}$ \\ evxlong173@163.com
}

\begin{abstract}
In this experiment, photocatalysic material, which is magnetic, photoluminesce and has superior light absorbing mesoporous, was synthesized by sol-gel method with tetrabutyl titanate、zinc acetate、stannic chloride antimonic chloride and ferric nitrate as precursors, and tri-blocked copolymer $\mathrm{F}_{127}$ as the template.Based on the experiment results, when the annealing temperature is at $550^{\circ} \mathrm{C}$, the annealing time is $120 \mathrm{~min}$, the doping amount of $\mathrm{Zn}$ is $4.0 \mathrm{at} \%$. These produced samples were shown to have superior luminescence and UV-vis light absorbance. When the weight of template was set to $1.5 \mathrm{~g}$, the obtained photocatalysic materials, indicated that their porous mainly distributed within $1.2 \mathrm{~nm}$ to $4.7 \mathrm{~nm}$ and their specific surface areas were larger than $110 \mathrm{~m}^{2} / \mathrm{g}$, and had excellent absorption properties.
\end{abstract}

Keywords: Three Dimensional Particle-Electrode, Process Condition, Structure, Optical Properties

\section{Introduction}

In recent years, micro-nanometer sized $\mathrm{TiO}_{2}$ hollow structures nanomaterials have attracted a great deal of attention because of their high photo-catalytic activity, chemical stability, low cost, nontoxicity, low density, high surface area, superior surface permeability and large light-harvesting efficiencies ${ }^{[1-6]}$. Moreover, these materials can be widely applied in photoelectric devices, catalysis, drug delivery, chromatography separation and chemical reactors $^{[7-12]}$. Various synthetic methods were explored to prepare hollow nanomaterials including self-assembly techniques, hydrothermal techniques, template-assisted techniques and chemically induced self-transformation ${ }^{[13-15]}$. Up to now, template-assisted synthetic method has been proved to be the most-applied and most-effective route to fabricate inorganic hollow structures. Titanium dioxide $\left(\mathrm{TiO}_{2}\right)$, as one of the most important transition-metal functional oxides, has attracted extensive attention during the past decades due to its superior physical and chemical properties and a wide variety of potential use in diverse fields such as solar energy conversion, environmental purification and water-treatment ${ }^{[16-18]}$. 
However, because of its wide band-gap energy $(3.02 \mathrm{eV}), \mathrm{TiO}_{2}$ can only harvest the spectrum with wave lengths in the near-ultraviolet (UV) region shorter than $387 \mathrm{~nm}$, which accounts for merely $4-5 \%$ of the solar spectrum. Moreover, $\mathrm{TiO}_{2}$ follows a relatively high electron-hole recombination rate, which is detrimental to its photo-activity. To solve this issue, different approaches such as transition metal doping, inorganic dye-sensitizing, valuable metal deposition and coupling titania with other semiconductors have been devoted to enhancing the photo-catalytic activity of $\mathrm{TiO}_{2}$ in which the response of the semiconductor was extended toward the visible region ${ }^{[19-21]}$.

Currently, it is still a great challenge to effectively immobilize or separate the $\mathrm{TiO}_{2}$ particle sin the photo-catalytic system. Magnetic separation provides a very convenient approach for removing and recycling magnetic particles/composites by applying an appropriate magnetic field ${ }^{[22,23]}$. Compared to conventional nano-powder photocatalysts, $\mathrm{TiO}_{2}$ magnetic composites can be regarded as a promising photocatalyst for the environmental purification at the industrial scale as they can be more readily separated from the slurry system by the magnetic separation after photocatalytic reaction and recycled.

Herein, novel $\mathrm{ZnO} / \mathrm{SnO} / \mathrm{SbO} / \mathrm{Fe}_{2} \mathrm{O}_{3} / \mathrm{TiO}_{2}$ magnetic meso-porous luminescence materials were successfully fabricated using Sol-Gel-template method. It has been carefully researched that the effects, which was caused by the annealing conditions and the doping amount of zine and tin, on the structure, absorption property, light absorbing and luminesce property of the catalytic materials. Furthermore, the influence of amount of template on the distribution of porous and absorption capacity has been discussed in this paper also.

\section{Experimental Procedures}

The preparation of sol A: The butyl titanate was dissolved in Ethyl Alcohol. The solution was moved to a Kjeldahl flask.Certain amount of additives and different amounts of zinc acetate were added into the flask. This mixture was stirred slowly by a magnetic stirrer for 2 hours until all components were dissolved completely. During this process the temperature was constant at $40{ }^{\circ} \mathrm{C}$. Then the transparent sol A was obtained and moved to a dry beaker.

The preparation of sol B: Ethyl Alcohol and Acetic acid were mixed up together and moved to a Kjeldahl flask. This solution was stirred by a magnetic stirrer and the temperature was kept in $40^{\circ} \mathrm{C}$. At the same time, sol A was dropped into the solution at the speed of 1 drop per second. After this, Macrogol 4000 was added into the flask. When the above solution was become clear, concentrated nitric acid was added slowly at the speed of 1 drop per 30 seconds. Then the PH value of the solution was adjusted to 3.0. This mixture was stirred continually for 3 hours, then sol B was gained which was transparent, light yellow.

Sol B was aged for 12 hours at the room temperature. Then sol B was put into a drying-oven for 240 minutes at the constant temperature $80^{\circ} \mathrm{C}$, then sol $\mathrm{B}$ was changed to semi-transparent gel. Subsequently, the temperature of drying-oven was increased to $105^{\circ} \mathrm{C}$ for another 240 minutes. Then sol B was become into solid powder and grounded in a star shape grinder for 30 minutes

The solid powder was moved into a crucible which was inside of a tube furnace. The temperature and time of anneal were stetted differently forgetting different samples. The sample was cooled down to room temperature naturally.

The XRD, UV-vis Absorption Spectrum, PL spectrum and BET surface area analyser were used to analyses and characterize the structure and property of the samples. 


\section{Results and Discussion}

\subsection{Effect of Zn-doped concentration on the structure and optical properties of three dimensional particle-electrode}

In this experiment, the contents of $\mathrm{Ti}, \mathrm{Sb}, \mathrm{Sn}$, and $\mathrm{Fe}$ were constant, the doping amount of $\mathrm{Zn}$ was changed, and the details were shown as $0 \%, 0.5 \%, 1 \%, 2 \%, 4 \%, 6 \%$ and $8 \%$ (percentage of $\mathrm{Zn}$ to $\mathrm{Ti}$ ). According to the method which was described above, the obtained dry solid powder was put into a tube furnace, the annealing temperature was at $550^{\circ} \mathrm{C}$ and the annealing time was set in 120 minutes.

\subsection{Structure Characterization}

Fig. 1 was the XRD spectrum of $\mathrm{Zn} / \mathrm{Fe} / \mathrm{Sn} / \mathrm{Sb}-\mathrm{TiO}_{2} 3 \mathrm{D}$ Particle Electrode with different doping amounts of $\mathrm{Zn}$. As shown in Figure 1, the crystal structures of samples have been deformed generally as increasing the doping amount of $\mathrm{Zn}$. When the doping amount of $\mathrm{Zn}$ was less than $4 \%$, the main diffraction peaks distributed at $2 \theta=25.68^{\circ}, 38.17^{\circ}, 48.32^{\circ}$, $55.37^{\circ}, 63.05^{\circ}$, the corresponding crystal surfaces of anatase crystal structure were 101,004 , 200, 211 and 204 respectively. When the doping amount of $\mathrm{Zn}$ was more than 8\%, the crystal structure of the samples changed significantly. The intensity of the diffraction peaks of rutile was increased, at the same time, the diffraction peak of $\mathrm{ZnTiO}_{3}$ and some unrecognized diffraction peaks have been observed.

\section{Optical Properties}

\subsection{PL Spectrum}

As shown in the Fig.2, during the wavelength was in the range of 300 to $375 \mathrm{~nm}$, every sample had a diffraction peak. As the wavelength range increased to $400-500 \mathrm{~nm}$, the diffraction peak of sample without Zn-doped (A1) disappeared. Those samples that included $\mathrm{Zn}$ component all had a diffraction peak, however their peak heights were different due to the amount of $\mathrm{Zn}$. The amount of $\mathrm{Zn}$ increased successively in alphabetical order from B to $\mathrm{G}$. The $\mathrm{Zn}$ content of sample E1 is $4 \%$, based on the Fig.2, it can be concluded the intensity of luminescence peak increased as the zine content increased when the doping amount of zine was lower than $4 \%$, when the doping amount of zine was higher than $4 \%$, the intensity of luminescence peak decreased as the zine content increased. When the doping amount of zine was equal to $4 \%$, the intensity of luminescence peak was the strongest and the width of FWHM became wider significantly.

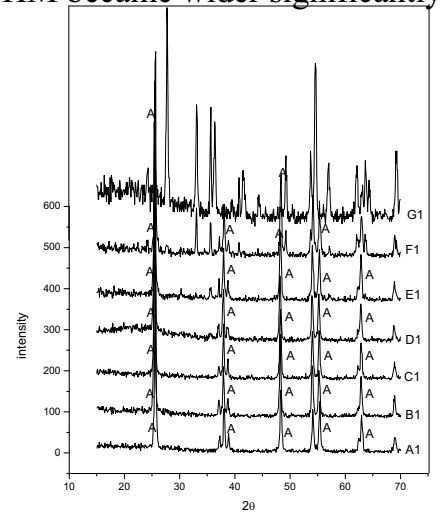

Fig.1 XRD Spectrum of the samples with different $\mathrm{Zn}$ contents (A1:0\%, B1:0.5\%, C1:1\%, D1:2\%, E1:4\%, F1:8\%, G1:16\%) 


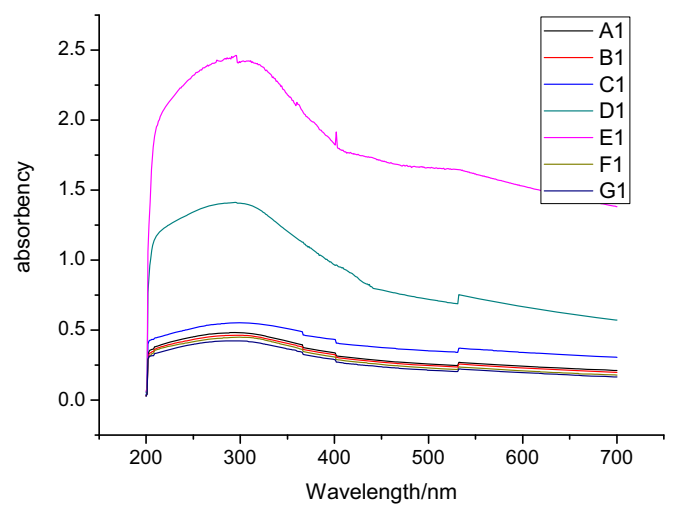

Fig.2 PL Spectrum of samples with different zine contents

\subsection{UV-vis Absorption Spectrum}

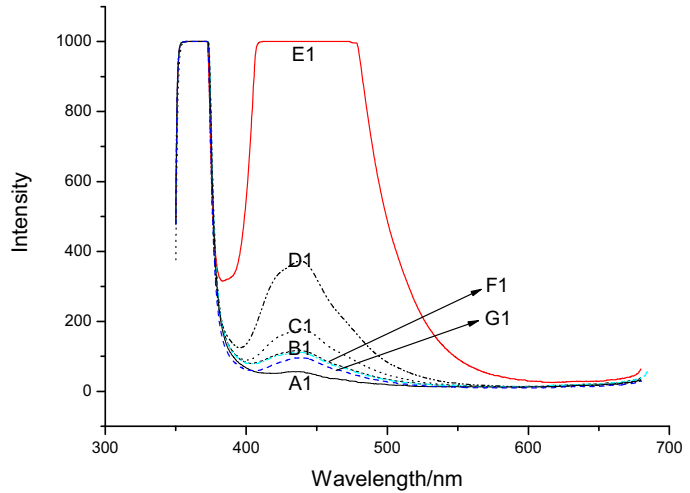

Fig.3 UV-vis Spectrum of samples with different zine contents

Based on the Fig.3, the absorption peaks of samples A1, B1, C1, F1 and G1 were quite weak at the ultraviolet region, these samples were hardly to absorb the light at visible region. On the contrary, samples D1 and E1 both had higher absorption peak at ultraviolet region and could absorb more light at the visible region. Especially, the capacity of absorbing light of sample E1 is distinct when the wavelength was between $200 \mathrm{~nm}$ to $700 \mathrm{~nm}$. For a photo catalysing material, excellent absorbance is one of the fundamental conditions to obtain the advantageous photo catalysing property.

\subsection{The influence of annealing Temperature and Time}

\subsubsection{The influence of annealing temperature}

The samples were annealed at $450^{\circ} \mathrm{C}, 550{ }^{\circ} \mathrm{C}, 600^{\circ} \mathrm{C}, 650^{\circ} \mathrm{C}$ and $700^{\circ} \mathrm{C}$ for $120 \mathrm{~min}$ respectively and then cooled down to room temperature.

When the temperature was higher than $600^{\circ} \mathrm{C}$, the intensity of diffraction peak of anatase structure reduced, the intensity of diffraction peak of rutile structure increased. Therefore, the annealing temperature should be lower than $600^{\circ} \mathrm{C}$. 


\subsubsection{The influence of annealing time}

The samples were annealed at $550{ }^{\circ} \mathrm{C}$ for $90 \mathrm{~min}, 120 \mathrm{~min}, 150 \mathrm{~min}$ and $180 \mathrm{~min}$, respectively and then cooled down to room temperature.

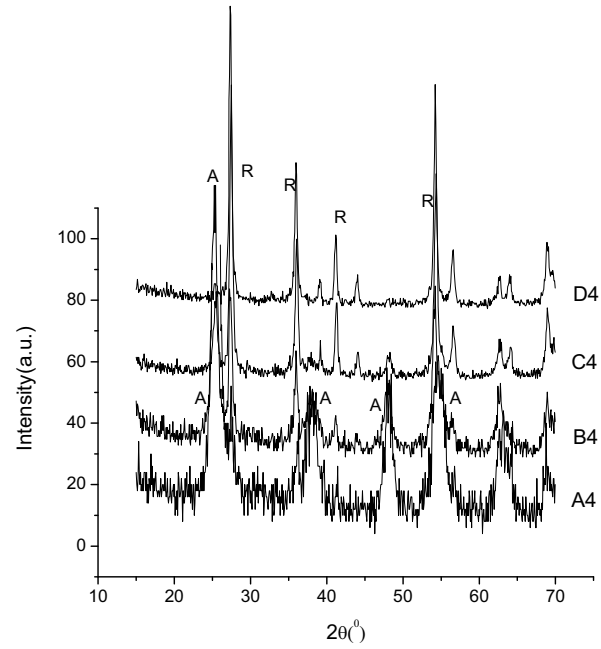

Fig.4 XRD spectrum of samples in different annealing temperature

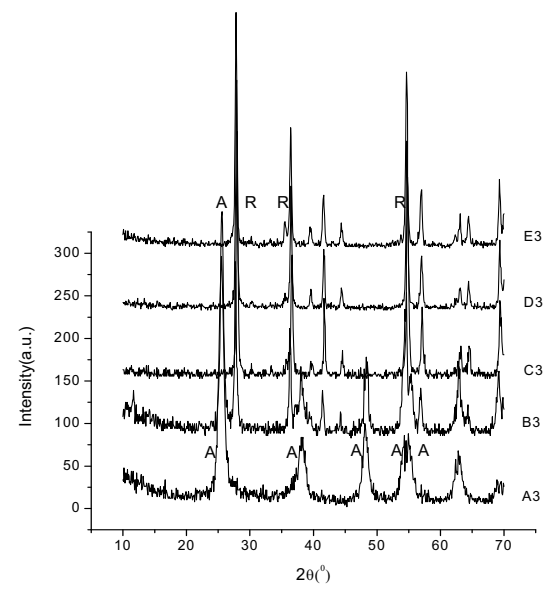

Fig.5 XRD spectrum of samples with different annealing time

According to the Fig.5, when the annealing time was 90 minutes, there was only one diffraction peak of anatase structure, but the peak height was low, the peak FWHM was wide. This indicated that short annealing time was not good at the growth of crystal. When the annealing time was increased to 120 minutes, the main diffraction peak was anatase structure peak, the intensity of diffraction was strong, the peak FWHM became narrow. This indicated that this annealing time was conductive to the growth of crystal. When the annealing time was more than 150 minutes, the intensity of diffraction peak of anatase structure decreased, on the contrary, the intensity of rutile structure increased. Therefore, the annealing time should not be more than 120 minutes.

\subsubsection{The influence of the amount of template on the absorbance of $\mathrm{Zn} / \mathrm{Fe} / \mathrm{Sn} / \mathrm{Sb}$-TiO2 3D Particle Electrode}

The amount of each element in the preparation of sol A and sol B was unchanged,as Zn4.0at\%,Sb1.0at\%,Sn2.0at\% and Fe2.5at\%, respectively. The amount of template F127 was $0 \mathrm{~g}, 0.5 \mathrm{~g}, 1.0 \mathrm{~g}, 1.5 \mathrm{~g}, 2.0 \mathrm{~g}, 2.5 \mathrm{~g}, 3.0 \mathrm{~g}$ and $3.5 \mathrm{~g}$.

From the Fig.6, it can be concluded in that the usage of template could improve the absorbance and specific surface area of electrode significantly. adsorption isotherm of samples all had regular hysteresis Loop, it indicated that samples had proper meso-porous structure. When the amount of template was lower than $1.5 \mathrm{~g}$, the specific surface area increased significantly as the amount of template increased, the peak width of pore-diameter distribution curve decreased significantly as the amount of template increased. When the amount of template was $1.5 \mathrm{~g}$, the corresponding specific surface area was $110 \mathrm{~m}^{2} / \mathrm{g}$, the peak width of the pore-diameter distribution curve reduced to $1.2-4.7 \mathrm{~nm}$. According to the calculation, the volume of pore, which was range at 1.2 to $4.7 \mathrm{~nm}$, was $84 \%$ of the total pore volume of the sample, it indicated that the samples that were made under this condition had excellent pore-diameter distribution. When the amount of template was more than $1.5 \mathrm{~g}$, the specific surface area of samples decreased as the amount of 
template increased, the peak width increased obviously as the amount of template increased. Therefore there was an optimum amount of template. Overloaded template would affect the pore-diameter distribution of the samples significantly, the quantity of the pore-diameter which was more than $10 \mathrm{~nm}$ increased distinctly. Therefore, when the amount of template was more than $1.5 \mathrm{~g}$, the specific surface area of samples decreased significantly as the template increased rather than increasing.

\section{Conclusion}

The novel $\mathrm{ZnO} / \mathrm{SnO} / \mathrm{SbO} / \mathrm{Fe}_{2} \mathrm{O}_{3} / \mathrm{TiO}_{2}$ magnetic meso-porous photo-catalytic materials were successfully fabricated by using Sol-Gel-template method. This experiment included the detailed investigation about the influence on the catalytic materials caused by changing annealing conditions and the doping amount of zine and tin. In addition, it also focused on the relationship of the porous distribution, absorption capacity and the amount of template.

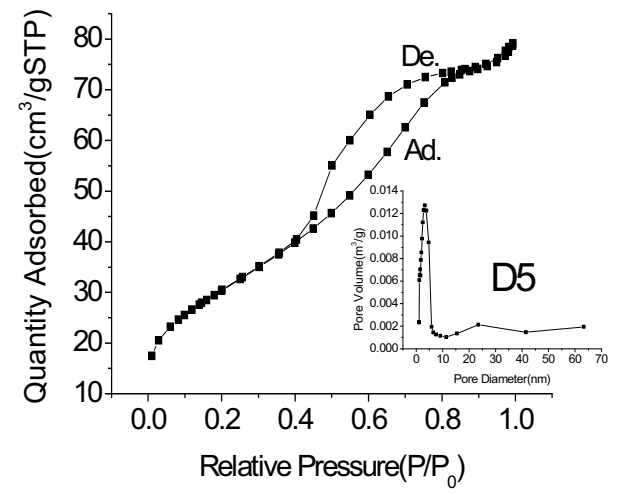

Fig.6 Porous distribution curve and adsorption isotherm of the sample(1.5g template)

When the annealing temperature was at $550^{\circ} \mathrm{C}$ and annealing time was $120 \mathrm{~min}$, the doping amount of $\mathrm{Zn}$ was $4.0 \mathrm{at} \%$, obtained samples has excellent luminesce property. For the wavelength is in the range of $200 \mathrm{~nm}$ to $700 \mathrm{~nm}$, the obtained samples has superior light absorbance. Excellent light absorbance is essential for a photocatalystic material to have good performance. Under the condition that the template was set to $1.5 \mathrm{~g}$, the obtained photocatalysic materials, indicated that their porous mainly distributed within $1.2 \mathrm{~nm}$ to $4.7 \mathrm{~nm}$ and their specific surface areas were larger than $110 \mathrm{~m}^{2} / \mathrm{g}$, and had excellent absorption properties.

\section{Acknowledgement}

The authors wish to acknowledge the financial support of the National Natural Science Foundation of China (no. 41271506) and the Science and Technology Development Project of Yantai(no.2014ZH076) .

\section{References}

1. Xiaoyan Zhang, Yujun Sun, Xiaoli Cui, Zhiyu Jiang. Carbon-incorporated $\mathrm{TiO}_{2}$ microspheres: facile flame assisted hydrolysis of tetrabutyl orthotitanate and photocatalytic hydrogen production, International Journal of Hydrogen Energy, 2012,37 
(2):1356-1365.

2. Qian Zhang, Wei Li, Shouxin Liu. Controlled fabrication of nano-sized $\mathrm{TiO}_{2}$ hollow sphere particles vivacid catalytic hydrolysis/hydrothermal treatment. Powder Technology, 2011,212(1):145-150.

3. Kwangjin An, Soon Gu Kwon, Mihyun Park, Hyon Bin Na, Sung-Il Baik, Jung Ho Yu, Dokyoon Kim, Jae Sung Son, Young Woon Kim, In Chan Song, Woo Kyung Moon, Hyun Min Park and Taeghwan Hyeon. Synthesis of uniform hollow oxide nanoparticles through nanoscale acidetching. Nano Letters, 2008,8:4252-4258.

4. Hualan Zhou, Zhong Zou, Sha Wu, Fangzhou Ge, Ying Li, Wenjian Shi. Rapid synthesis of $\mathrm{TiO}_{2}$ hollow nanostructures with crystallized walls by using $\mathrm{CuO}$ as template and microwave heating. Material Letters, 2011,65(6):1034-1036.

5. Jiaguo Yu, Shengwei Liu, Huogen Yu. Micro structures and photo-activity of mesoporous anatase hollow micro spheres fabricated by fluoride-mediated self-transformation, Journal of Catalysis, 2007,249(1):59-66.

6. Sihui Zhan, Jiangyao Yang, Yu Liu, Nan Wang, Jingjing Dai, Hongbing Yu, Xichao Gao, Yi Li. MesoporousFe $\mathrm{O}_{3}$-doped $\mathrm{TiO}_{2}$ nanostructured fibers with higher photocatalytic activity, Journal of Colloid and Interface Science, 2011,355(2): 328-333.

7. Qinghang He, Zhenxi Zhang, Jianwen Xiong, Yuying Xiong, Hua Xiao. A novel biomaterial- $\mathrm{Fe}_{3} \mathrm{O}_{4}$ : $\mathrm{TiO}_{2}$ core-shell nano-particles with magnetic performance and high visible light photocatalytic activity. Optical Materials,2008,31(2):380-384.

8. Penghui Zhao, Weilong Li, Gang Wang, Baozhi Yu, Xiaojun Li,Jintao Bai,Zhaoyu Ren. Facile hydrothermal fabrication of nitrogen-doped graphene/ $\mathrm{Fe}_{2} \mathrm{O}_{3}$ composites as high performance electrode materials for supercapacitor. Journal of Alloys and Compounds. 2014,604:87-93.

9. Zhengying Gu, Xiangdong Gao, Xiaomin Li, Zhengwu Jiang, Yudi Huang. Nanoporous $\mathrm{TiO}_{2}$ aerogel blocking layer with enhanced efficiency for dye-sensitized solar cells. Journal of Alloys and Compounds. 2014,590:33-40.

10. Xue Bai, Xiaoyuan Zhang, Zulin Hua, Wenqiang Ma, Zhangyan Dai, Xin Huang, Haixin Gu. Uniformly distributed anatase $\mathrm{TiO}_{2}$ nanoparticles on graphene: Synthesis, characterization, and photocatalytic application. Journal of Alloys and Compounds,2014,599:10-18.

11. Guidong Yang, Zifeng Yan, Tiancun Xiao, Bolun Yang. Low-temperature synthesis of alkalis doped $\mathrm{TiO}_{2}$ photocatalysts and their photocatalytic performance for degradation of methyl orange. Journal of Alloys and Compounds,2013,580:15-22.

12. Mukhtar Ahmad, Ihsan Ali, R. Grössinger, M. Kriegisch, F. Kubel,M.U. Rana. Effects of divalent ions substitution on the microstructure, magnetic and electromagnetic parameters of $\mathrm{Co}_{2} \mathrm{~W}$ hexagonal ferrites synthesized by sol-gel method. Journal of Alloys and Compounds, 2013,579:57-64.

13. Xujie Lü, Fuqiang Huang, Jianjun Wu, Shangjun Ding, and Fangfang Xu. Intelligent hydrated-sulfate template assisted preparation of nanoporous $\mathrm{TiO}_{2}$ spheres and their visible-light application. ACS Applied Materials Interaces, 2011,3(2):566-572.

14. Jiaguo Yu and Xiaoxiao Yu. Hydrothermal synthesis and photocatalytic activity of zinc oxide hollow spheres. Environmental Science Technology, 2008,42(13) 4902-4907. 
15. Xiaoning Wang, Baibiao Huang, Zeyan Wang, Xiaoyan Qin, Xiaoyang Zhang, Ying Dai and Myung-Hwan Whangbo. Synthesis of anatase $\mathrm{TiO}_{2}$ tubular structures microcrystallites with a high percentage of $\{001\}$ facets by a simple one-step hydrothermal template process, Chemistry European Journal, 2010,16(24):7106-7109.

16. Bin Liu and Eray S. Aydil. Growth of oriented single-crystalline rutile $\mathrm{TiO}_{2}$ nanorods on transparent conducting substrates for dye-sensitized solar cells. Journal of the American Chemical Society. 2009,131(11):3985-3990.

17. Yunqian Dai, Claire M. Cobley, Jie Zeng, Yueming Sun and Younan Xia. Synthesis of anatase $\mathrm{TiO}_{2}$ nanocrystals with exposed $\{001\}$ facets, Nano Letters. 2009,9(6)2455-2459.

18. Haifeng Lin, Liping Li, Minglei Zhao, Xinsong Huang, Xiaomei Chen, Guangshe Li, and Richeng Yu. Synthesis of high-quality brookite $\mathrm{TiO}_{2}$ single-crystalline nano sheets with specific facet sexposed: tuning catalysts from inert to highly reactive. Journal of the American Chemical Society, 2012,134 (20):8328-8331.

19. Azrina Abd Aziz, Kok Soon Yong, Shaliza Ibrahim, Saravanan Pichiah. Enhanced magnetic separation and photocatalytic activity of nitrogen doped titania photocatalyst supported on strontium ferrite. Journal of Hazardous Material, 2012,199-200:143-150.

20. Jean-Marie Herrmann. Titania-based true heterogeneous photocatalysis. Environmental Science and Pollution Research, 2012,19(9)3655-3665.

21. Jean-Marie Herrmann. Detrimental cationic doping of titania in photocatalysis: why chromium $\mathrm{Cr} 3+$-doping is a catastrophe for photocatalysis both under $\mathrm{UV}$ - and visible irradiations. New Journal of Chemistry, 2012,36(4): 883-890.

22. Raquel V. Mambrini, Thales L. Fonseca, Anderson Dias, Luiz C.A. Oliveira, Maria Helena Araujo, Flávia C.C. Moura. Magnetic composites based on metallic nickel and molybdenum carbide: a potential material for pollutants removal. Journal of Hazardous Material, 2012,241-242: 73-81.

23. Qin Li, Hui Li, Runming Wang, Guangfang Li, Hao Yang, Rong Chen. Controllable microwave and ultrasonic wave combined synthesis of $\mathrm{ZnO}$ micro-/nanostructures in HEPES solution and their shape-dependent photocatalytic activities. Journal of Alloys and Compounds, 2013,567:1-9. 